\title{
Progress of ESO's 100-m OWL optical telescope design
}

\author{
E. Brunetto, P. Dierickx, R. Gilmozzi, M. Le Louarn, \\ F. Koch, L. Noethe, Ch. Vérinaud, N. Yaitskova
}

European Southern Observatory

\begin{abstract}
Even as a number of 8 - to 10 -m class telescopes come into operation worldwide, the scientific challenges these instruments and their space-based counterparts already address imply that future increases in lightgathering power and resolution will have to exceed conventional scaling factors. Indeed, it can be exp ected that the same progress in telescope diameter and resolution achieved throughout the century must now be realized within, at most, one or two decades. The technologies required to assert the validity of such an extrapolation appear to be within reach. Large telescopes successfully commissioned within the last decade have demonstrated key technologies such as active optics and segmentation. Furthermore, current design methods and fabrication processes imply that the technological challenge of constructing telescopes up to the 100- $\mathrm{m}$ range could, in some critical areas, be lower than those underlying, two decades ago, the design and construction of 8 to $10-\mathrm{m}$ class telescopes. At system level, however, such giants are no size-extrapolated fusion of VLT and Keck, but fully integrated adaptive systems. In this paper we elaborate on some of the science drivers behind the OWL concept of a $100 \mathrm{~m}$ telescope with integrated adaptive optics capability. We identify major conceptual differences with classical, non-adaptive telescopes, and derive design drivers accordingly. We also discuss critical system and fabrication aspects, and the possible timeline for the concept to be realized.
\end{abstract}

\section{INTRODUCTION}

After preliminary work had hinted that giant telescopes might be within the reach of existing technology [1], in 2000 ESO started a phase A study for a 100-m class optical telescope, dubbed OWL for its keen night vision and for being OverWhelmingly Large. Proposals for telescopes with diameters much larger than deemed possible had been laid down as early as 1978 [2,3], 1992 [4] and 1996 [5]. Building on the VLT experience and on dedicated industrial studies, ESO subsequently undertook the design of an even more ambitious, 100-m concept. Driven by a powerful science case, i.e. the search for signs of life in extra-solar earth-like planets, the size of the OWL telescope is just above the minimum necessary to achieve the goal of obtaining spectroscopy of potential targets around the nearest stars $(\sim 80-\mathrm{m})$, and opens the way to observational possibilities unheard of until now.

\begin{tabular}{|c|c|}
\hline Optics & $\begin{array}{l}\text { 6-mirror, } \mathrm{f} / 7.5, \sim 6,900 \mathrm{~m}^{2} \text { collecting area, near-circular outer rim } \\
\text { Spherical dia. } 100 \mathrm{~m}, \mathrm{f} / 1.2\end{array}$ \\
\hline \multicolumn{2}{|r|}{. } \\
\hline M2 & Flat, dia. $25.6 \mathrm{~m}$ \\
\hline Corrector & 4 elements, dia. $8,8,3.5,2 \mathrm{~m}$ \\
\hline FOV & $\begin{array}{l}10 \text { arc min. seeing-limited; } 6 \text { focal stations (rotation of M6) } \\
>2 \text { arc min.diffraction-limited (vis.) }\end{array}$ \\
\hline Structure & $\begin{array}{l}2.1 \mathrm{~Hz} \text { eigenfrequency, } 14,200 \text { tons moving mass; } \\
\text { Standard steel beams, serially produced joints; }\end{array}$ \\
\hline Kinematics & Distributed low-cost friction drives, selfadjusting; \\
\hline Control & $\begin{array}{l}\text { Multi-stage, distributed wavefront control: phasing, pre-setting, field } \\
\text { stabilization, focusing, fine centering, dual conjugated active optics, adaptive } \\
\text { optics }\end{array}$ \\
\hline
\end{tabular}

Table 1. Owl overall characteristics. 
Bar adaptive optics, the feasibility of the crucial components of a giant telescope is no longer questioned. All industrial studies commissioned by ESO within the framework of its OWL design study confirm that the technological risk underlying the production of thousands of segments and structural elements is intrinsically lower than the risks taken two decades ago with 8- to 10 -m class telescopes. Feasibility per se is evidently not sufficient; novel design approaches are required to guarantee cost-effective, streamlined production, integration and maintenance of the subsystems, and to ensure that the telescope works as a coherent and reliable system.

The design of the Owl 100-m telescope relies extensively on proven fabrication technologies, in particular on mass- or serial-production schemes, and incorporates several distinct wavefront control loops. The overall characteristics of the current design are listed in table 1.

\section{SCIENCE WITH OWL}

A 100 -m telescope working at the diffraction limit (e.g. 1 mas at V 37) has an unprecedented scientific potential (not least for new discoveries). Here we summarize some recently developed science cases for OWL, in particular those that take full advantage of its capabilities: the spectroscopy of exo-earths to look for signs of life, and some of the cosmological science cases. Before we do that, there are two general aspects about the scientific performance of ELTs that deserve some comments:

Confusion about confusion. There is a widespread concern that ELTs may hit the confusion limit, thereby voiding their very raison d'être. Much of this concern comes from past observations at poor angular resolution (e.g. X-ray data or deep optical images in 2" seeing of the '80s). Recent results with better resolution lead to resolving the "confusion" into individual objects (e.g. the X-ray background, now mostly if not completely resolved, or the HDF images showing 20 times more empty space than objects). Ultimately, some confusion level will be reached, but the 3-dimensional nature of astronomical objects (position and velocity) virtually ensures it will not be a limiting factor with OWL. In fact, a lack of confusion may offer information on the covering factor of galaxies, and seems tantalizingly connected to Olbers' paradox.

Signal-to-noise vs diameter D. A common misapprehension regards the dependence of signal $\mathrm{S}$ and signal-to-noise $\mathrm{S} / \mathrm{N}=\mathrm{S} / \sqrt{ }\left(\mathrm{S}+\mathrm{Bgd} * \mathrm{n}_{\text {pix }} *\right.$ pixsize $\left.+\mathrm{n}_{\text {pix }} * \mathrm{RN}^{2}\right)$ on $\mathrm{D}$ when the telescope works at the diffraction limit. Too often one finds an $S \propto D^{4}$ assumption which is (unfortunately!) not true: while the peak of the PSF indeed increases as $D^{4}$, its integral within a typical $\lambda \mathrm{D}$ pixel increases as $\mathrm{D}^{2}$. This means that the $\mathrm{S} / \mathrm{N}$ is proportional to $\mathrm{D}^{2}$ in the backgroundlimited regime $\left(S \propto D^{2}, B g d \propto D^{2}\right.$, pixsize $\propto D^{-2}, S / N \propto D^{2} / \sqrt{ }$ const $)$, and to $D$ in the shot noise regime $\left(S / N \propto D^{2} / \sqrt{ } D^{2}\right)$. The time to achieve the same $\mathrm{S} / \mathrm{N}$ for different telescope sizes is $\propto(\mathrm{S} / \mathrm{N})^{2}$ and is a better estimator of the relative performance of different telescope diameters.

Spectroscopy of earth-like exoplanets. Searching for exo-biospheres could be defined as the holy grail of today's astronomy. This science case depends very strongly on the telescope diameter. First of all, the volume accessible to a telescope is proportional to $\mathrm{D}^{3}$ (since resolution is $\propto \mathrm{D}$ ). For example, the number of accessible $\mathrm{G}$ stars in the solar neighbourhood where an earth at $1 \mathrm{AU}$ could be detected would be 20, 165 and 750 respectively for a 30-, 50- and 100$\mathrm{m}$ telescope (assuming minimum resolvable separation 5//D). From the point of view of the sensitivity, the time to achieve the same $\mathrm{S} / \mathrm{N}$ in the planet (for objects in common, of course) is $\propto \mathrm{D}^{4}$ (the planet is $\sim 10^{10}$ times fainter than the parent star, and even at many $\lambda D$ from the star we are definitely in the background-limited regime). This means that a 30-m telescope would need $\sim 120$ times longer than OWL to detect the same object. Our simulations indicate that for a solar system analogue at $10 \mathrm{pc}$ the earth would be detected in about one hour with OWL, opening up the possibility of doing spectroscopy for D $>80 \mathrm{~m}$. A recent study by Angel (2003) confirmed that spectroscopy of exo-earths is within the reach of a $100-\mathrm{m}$ in the optical/near-IR, while in the thermal infrared space interferometers (à la Darwin/TPF) are moderately better unless the $100-\mathrm{m}$ is placed in Antarctica. Sophisticated corona graphic techniques, able to suppress by factors upward of $10^{6}$ the light of the parent star may allow to resolve closer separations, at the same time expanding the accessible volume and decreasing the necessary exposure times.

Measure of cosmological parameters with primary distance indicators (note: H, not Hnot!) Beyond the local universe, distances are determined today using derived standard candles (e.g. SNe Ia) whose calibration is not always 
completely reliable. OWL will allow the measurement of distances using primary indicators in the redshift range where the difference between cosmological models is more pronounced. Using Cepheids and Novae, as well as the planetary nebulae and globular clusters luminosity functions, accurate measurements of distances can be obtained out to $\mathrm{z} \sim 1$ and these will allow to disentangle the various models, including those alternative to $\Lambda$. Beyond $\mathrm{z} \sim 1$, Type Ia SNe can be detected up to $\mathrm{z} \sim 5$ (although Ia's are the second brightest explosions in he universe after GRBs they do not emit in the $\mathrm{UV}$ and $\mathrm{K}$ being the last useful band they are not detected beyond that $\mathrm{z}$ ), core-collapse $\mathrm{SNe}$ out to $\mathrm{z} \sim 10$, and alleged Pop III SNe even beyond. While SNe may be more useful to determine the cosmic supernova rate, and from this the early universe star formation history, than in disentangling cosmologies, they will provide critical cosmological information up to redshift $\sim 10$ (e.g. quintessence?).

Primordial stellar populations. WMAP sets the recombination epoch at $\mathrm{z}$ between 10 and 20. Pop III stars are strong candidates as possible re-ionization sources. Pop III stars are hot and massive, and form before re-ionization possibly in dwarf-galaxy sized over-densities $\left(10^{6}-10^{7} \mathrm{M} \odot\right)$. Strong emission lines of $\mathrm{H}$ and $\mathrm{He}$, and strong nebular continuum characterize their spectra. Both the continuum and line spectrum can accurately be studied with OWL at high $\mathrm{z}$, thus detecting and characterizing the first-light objects in the Universe. OWL would be about 10 times more efficient than JWST for these observations.

\section{OPTICAL DESIGN}

The question of optical design is the subject of lively discussions. In theory, the Ritchey -Chrétien design can hardly be surpassed, as it provides a relatively large field of view and a minimum number of reflections, hence minim al emissivity in the infrared. So far discussions have focused on the segments fabrication issue, which can only be part of a more complex trade-off at system level. A large telescope is a controlled opto-mechanical assembly; the consequences of optical solutions cannot be narrowed down to the sole fabrication of the segments. At an earlier stage of the OWL study, the ESO has evaluated a fairly broad range of opto-mechanical designs [6] in relation to cost, risk, feasibility, performance, maintainability and operations. These evaluations consistently pointed towards spherical primary solutions, in view of the sheer size of the telescope and its exposure to inevitable excitations (gravity, wind, thermal change), its versatility, functional and performance requirements, and its cost ceiling (1 billion Euros in capital investment).

Still, the discussion is not closed, and will continue producing fruitful results, with proponents of different designs working hard on developing cost-effective technologies supporting their designs. In brief, ESO's decision to opt for a baseline spherical primary mirror solution is, ironically but very usefully, leading to substantial technology development in rival aspherical segments production technologies, and subsequent cost reduction. Hard numbers are still missing; according to informal discussions with industrial suppliers, the cost increase from spherical to aspherical segments may have gone, in the past five years, from a factor 4-5 to less than two.

The OWL optical design is shown in figure 1. It is based on a spherical and flat folding secondary mirrors, with a four element corrector providing for the compensation of spherical and field aberrations as well as advanced active optics and dual-conjugate adaptive optics. A complete discussion would exceed the scope of this report; we shall however mention a few key arguments supporting this solution:

- Low sensitivity to lateral decenters (hence to gravity, wind and thermal excitations), the alignment of the primary and secondary mirrors being quite inconsequential, and stiffness at the location of the critical subsystem (the corrector) being fairly high ${ }^{1}$;

- Low number of surfaces (6) for the complete range of wavefront control functions: field stabilization, active focusing and centering, actively deformable surfaces, dual conjugated adaptive optics;

- Low cost and fabrication risk for long-lead items (primary mirror segments);

- Ease of integration and maintenance; interchangeability of all segments);

- Large, well-corrected field of view (10 arc minutes seeing-limited, 2 arc minutes diffraction -limited in the visible ${ }^{2}$ );

\footnotetext{
${ }^{1}$ Tolerances at the level of the corrector are comparable to those applying to the VLT $8 \mathrm{~m}$ telescopes, and easier to achieve in view of the increased design space. It shall be noted that in view of the size, and inevitable exposure to wind, sensitivity to wind excitation is of crucial importance.
} 
- Baffling, reduction of stray light implied by the availability of intermediate foci and pupil images;

- Availability of an intermediate focus (after the quaternary mirror) for the calibration of adaptive optics interaction matrices.

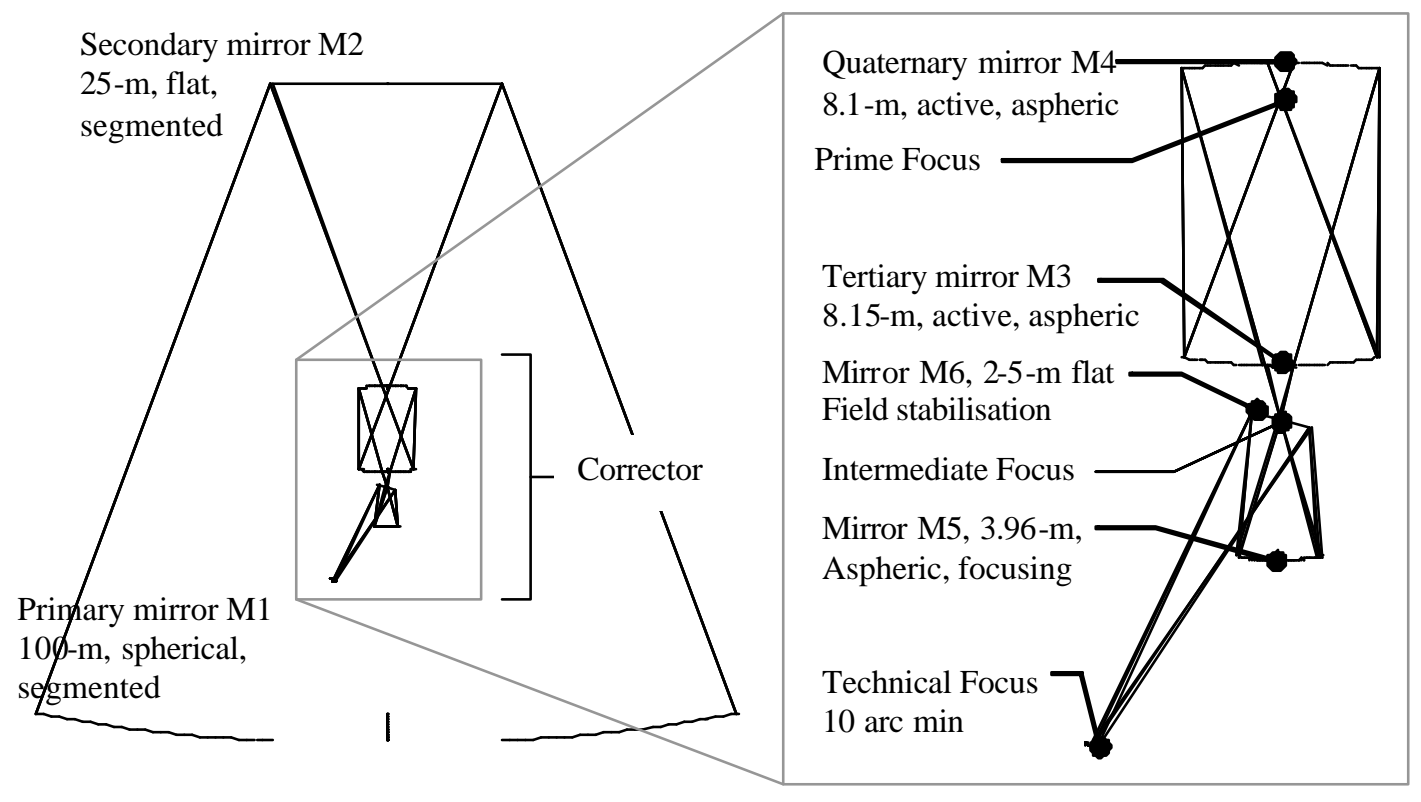

Fig. 1. Owl optical design layout

The main disadvantages of the design are the number of reflections, the implied emissivity ${ }^{3}$, and the difficulty to manufacture the quaternary mirror ${ }^{4}$. The properties of the design, including its ability to rely on Laser Guide Stars (LGS), have been extensively described in the literature and will not be recalled herein $[6,7,8]$.

\section{OPTICAL FABRICATION}

The very first question that comes to mind when dealing with giant telescopes is the cost-effective feasibility of its optics. Assuming classical materials for the segments blanks, however, there is no need for a very substantial increase in production capacity from existing suppliers ${ }^{5}$, provided that the segment size ${ }^{6}$ remains

below 2-m. Moderately lightweight Silicon Carbide is also considered as a serious and potentially cost-effective candidate, for its superior thermal performance and specific stiffness.

\footnotetext{
${ }^{2}$ For comparison, a classical Ritchey-Chrétien solution of similar dimensions would offer about 20 arc seconds diffraction-limited field of view.

${ }^{3}$ For IR applications demanding a very small field of view only, this disadvantage could be mitigated by exchanging the corrector for a simpler, single surface one, thereby limiting the number of hot surfaces to 3 . At the time of writing of this article, this option is however at a notional stage only.

${ }^{4}$ In the design presented here, the compensation of the enormous spherical aberration of the primary mirror inevitably falls on the quaternary one. With a f/1.42 spherical primary mirror, the aspheric departure to be figured into the quaternary is as large as $14 \mathrm{~mm}$.

5 This statement is supported by contracts placed by ESO with CORNING, SCHOTT, and LZOS for the serial production of segments blanks in ULE (Ultra-Low Expansion glass), Zerodur and Astro-Sitall, respectively.

${ }^{6}$ Another compelling reason to limit segment size is the cost of transportation; beyond 2.3 -m flat-to-flat segment size, transport in standard $40 \mathrm{ft}$ containers is excluded and transport costs increase beyond reasonable limits.
} 
With spherical segments, optical figuring and testing is a proven and reliable process, well suited for mass-production. Serial production of diffraction-limited, large optics is already under way for laser fusion projects, with European suppliers increasing their capacity to approximately $1,000 \mathrm{~m}^{2}$ per year ${ }^{7}$. Aspherical segments would certainly be feasible as well, but the inherent risk and potentially lower quality need to be properly evaluated. In figuring optical surfaces, polishing and testing play equally important roles. The final shape is basically determined by two references: the figuring tool and the metrology. Spherical segments can be polished with large-size, stiff tools, which provide a stable, passive reference, and tested against a unique matrix in a Fizeau interferometric arrangement. Aspherical segments, on the contrary, require that either the tool be flexible or small to accommodate for the aspheric departure, or that the part be bent with a dedicated warping harness and polished spherical, the aspheric shape being then atained when the bending moments are removed. In both cases the passive reference is lost, either because of the flexibility or small size of the tools, or because of residual internal stresses in the segment ${ }^{8}$. Metrology is an issue as well, the aspheric departure of individual segment, hence the characteristics of the metrology setup, being dependent of the segment's radial position. In brief, stringent quality control requirements and potentially lower yield make aspherical segments illadapted to cost-effective serial, reliable production.

As for the Owl 100-m telescope, the segments size (primary and secondary mirrors) is in the range 1.3-2.3-m flat-to-flat with a likely cost optimum at $1.6-\mathrm{m}$, corresponding to about 3000 segments for the primary and 250 for the secondary mirrors. The upper limit is set by the compatibility with cost-effective transport in standard containers. The lower limit, somewhat less clearly defined, is set by the implied cost and complexity of the control system. Two possible technologies are considered for the optical figuring: planetary polishing, and individual computer-controlled robots equipped with large, stiff tools. Replication has been briefly considered but rejected in view of the limited master durability and unpredictable surface stresses in the epoxy layer.

Planetary polishers, possibly complemented by local corrections with ion-beam or computer controlled, small tool polishers for final touches, seem ideal in view of the relative stability and high yield of the process. In this scheme, three to four 8- m class machines used serially would be required to achieve the required yield of about 1.5 segments per day.

Planetary polishers are however less attractive with large segments as the cost of large machines may become higher than that of a sufficiently large number of classical robots working parallel one piece at a time.

Besides the increase of reflections implied by Owl's optical design, a price to pay for the spherical primary mirror solution is the difficulty to compensate for its spherical aberration, and in particular the horrendous aspherization of the quaternary mirror (which is conjugated to the primary). A possible test setup has been identified and the state of current technology allows for cautious hope $\mathrm{e}^{8}$ industrial studies are however still required to confirm feasibility and evaluate implied cost and schedule.

\section{OPTO -MECHANICAL DESIGN}

The telescope has been designed as a coherent opto-mechanical system to ensure a reasonable balance between constraints. In particular, the optical solution has been tailored to avoid critical centering tolerances at locations where the structural design could not realistically provide high stiffness. Hence the use of a flat secondary mirror, a rather inefficient feature in terms of optical design, but an ideal one in relation to decenters. Critical tolerances inevitably show up, but they are now applying to the surfaces within the corrector, and to a lesser extent to the corrector itself. High structural stiffness is rather easily achieved at these locations, and the tolerances turn out to be comparable to those applying to ESO's Very Large Telescope (VLT), while allocations for design space and mass are far more generous with Owl.

Owl's opto-mechanical design is the result of ext ensive trade-offs between optical and structural considerations spanning over a wide range of potential solutions and involving cost, feasibility, performance, maintenance and operability criteria. For a structure of its size, its performance in terms of stiffness and mass is already quite impressive:

\footnotetext{
${ }^{7}$ A driving factor is the production of $\sim 600 \mathrm{~mm}$ amplifier plates for the Megajoule project.

${ }^{8}$ This problem can be alleviated by stringent requirements on the residual internal stresses of the segment blanks, however at a higher cost of the substrates.
} 
a locked rotor eigenfrequency of $2.1 \mathrm{~Hz}$, i.e. comparable to that of existing, much smaller telescopes, and a moving mass of "only" 14,000 tons. In comparison, the 8-m VLT scaled to Owl dimensions would be near ing 1,000,000 tons. In addition, Owl's structure is made of standard steel beams and serially produced nodes, thereby allowing low cost, fast supply and integration schemes. The alt-az design has been aptly named "fractal", with a six-fold symmetry and substructures cloned from main structural modules at scales that are integer multiples of the segment size. As a result, the design ensures ideal, nearly all-identical interfaces to the main optics, optimal load transfers, ideal maintainability and low cost, in addition to a near-circular pupil and a Point Spread Function exclusively dominated by 6-fold symmetry features.
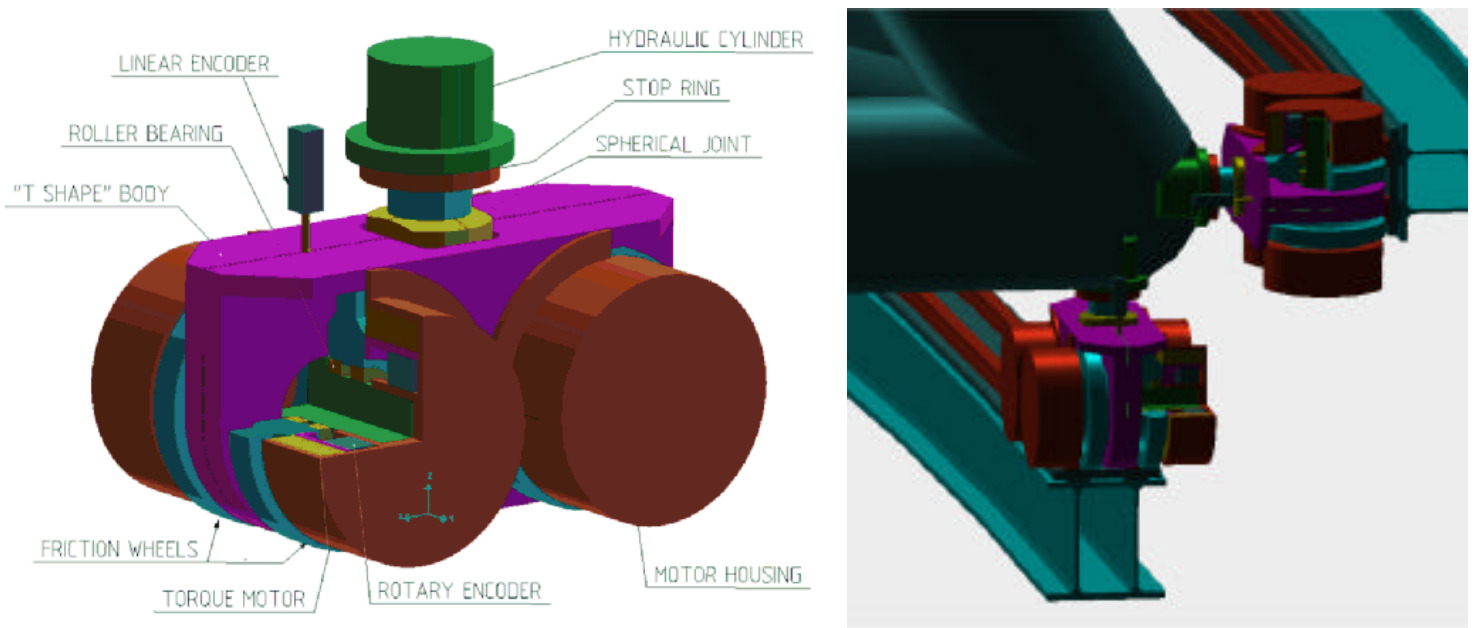

Fig. 3. Friction drives.

The design is supported by stress, fatigue, buckling and safety analysis, and relies on standard steel. Only in localized areas would higher quality (albeit still standard) steel be needed. Aramid ropes (Kevlar) instead of steel pre-tensioning cables may provide higher performance at a marginal cost increase.

The kinematics is provided by friction drives (bogies); hydrostatic bearings and direct drives have been briefly considered but the cost and complexity implied by kilometers of high-accuracy tracks were deemed unacceptable. ESO's experience with the VLT Coudé rotating platforms clearly showed that micron-accuracy could be readily achieved with properly designed friction drives and bearings. Figure 3 shows a notional design of Owl's friction drives and bearings and their interfacing to tracks. Several hundreds of individual units would be required, but the cost is modest in comparison to that of hydrostatic bearings and direct drives, with a far better load distribution hence relaxed foundation and alignment requirements. Hydraulic connections between individual bogies are foreseen as a way to periodically re-adjust load distributions to cope e.g. with seasonal changes.

A possible layout of the facility is shown in figure 4. The telescope would be operated in open air, with a low-cost sliding enclosure to protect it when not in operation. Computer Fluid Dynamics (CFD) simulations, measurements on radiotelescopes and wind tunnel testing are planned to verify that the range and bandwidth of the kinematics and wavefront control systems are sufficient to cope with wind buffeting in the structures and on the mirrors. The design being insensitive to lateral primary-secondary mirror decenters, and the corrector being located in a stiff area, the crucial issue is less tracking performance than rapidly varying deformations of the segmented mirrors. A preliminary CFD analysis has shown that quasi-static $(<1 \mathrm{~Hz})$ errors would be well within the range and bandwidth of the active optics and field stabilization control systems. At higher frequencies, the results are suspiciously optimistic, and can no longer be relied on - the complexity of a sufficiently high-resolution full-scale CFD model becoming unreasonably high.

The sliding enclosure concept is based on the design of an existing hangar built by SIAT South of Berlin to house giant Zeppelins (SIAT's hangar could accommodate two Owls). It was erected in about 1.5 years at a reported cost of 70 M€. In spite of being smaller, Owl's enclosure may not be significantly cheaper as more demanding site-dependent constraints may apply. Owl enclosure would provide passive thermal cooling only; bcal air conditioning is foreseen in 
primary and secondary mirror covers as well as in the corrector and the 6 instruments racks. The primary mirror covers, retracted from the telescope and stacked on top of each other, can be seen on figure 4 , in front of the enclosure.

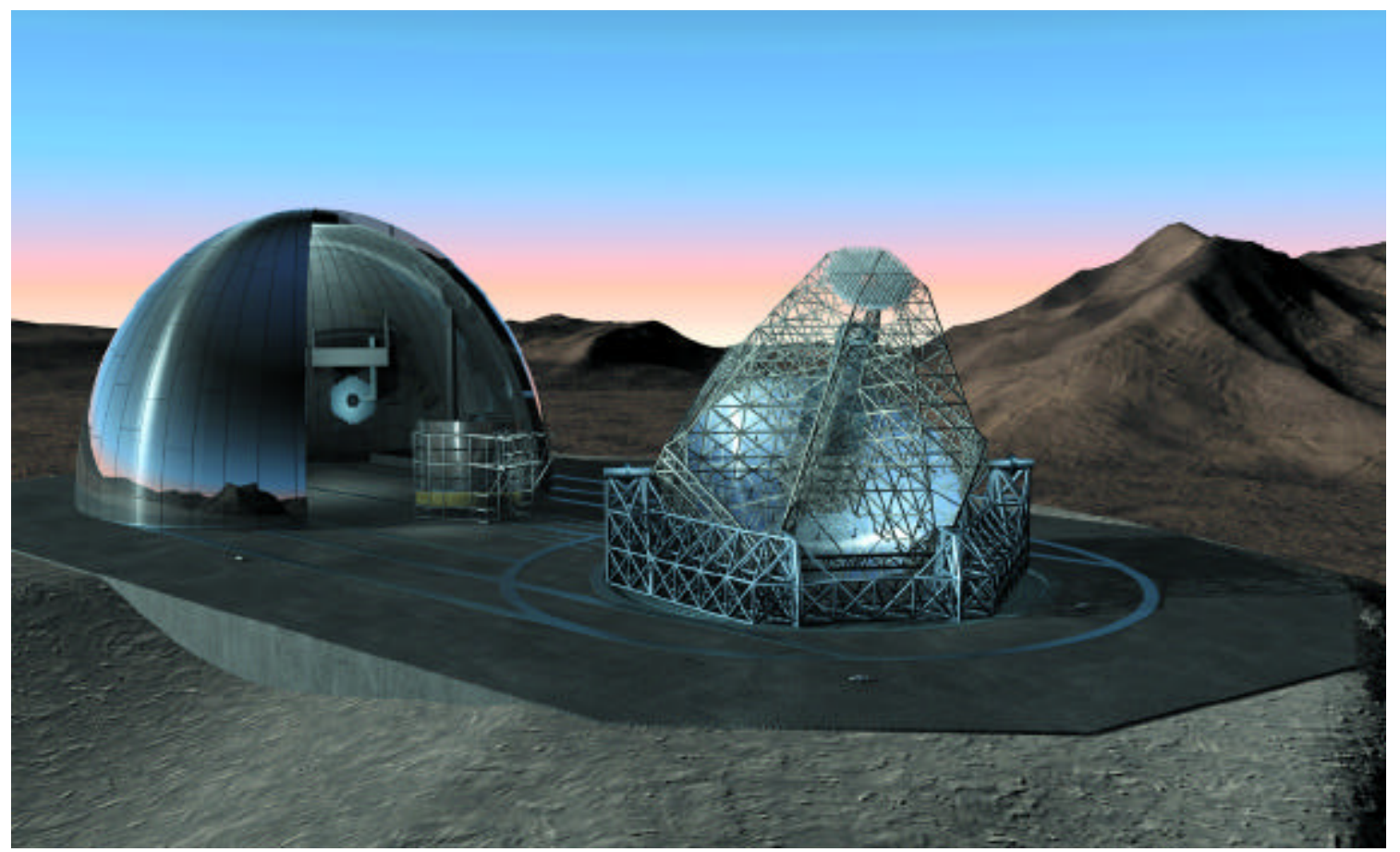

Fig. 4. Layout of the Owl observatory.

\section{CONTROL SYSTEMS}

The OWL concept is built on the VLT experience, with extensive wavefront control capability. The number of degrees of freedom and the implied complexity is however substantially larger, as shown in table 2 .

The first stage of wavefront control, pre-setting, is aimed at ensuring that optical surfaces are centered to an accuracy allowing subsequent loops to be closed reliably; in practice this means that the corrector shall be centered to a few mm accuracy, and surfaces within the corrector to typically $0.1 \mathrm{~mm}$ and about one arc second. Neither the metrology nor the actuation mechanisms required to achieve such tolerances do represent substantial challenges; a system currently being implemented in the South African Large Telescope (SALT) would most likely meet requirements, and an alternative solution based on fiber extensiometers will be evaluated in-situ in existing facilities.

Phasing of the segmented mirrors is a more complex issue, with several thousands of segments to be kept aligned within a few nm. The baseline solution is to mount capacitive sensors between segments to allow real -time measurement of the differential piston between adjacent segments. Noise propagation is well characterized, thanks to the Keck experience. Periodic on-sky calibration of the sensors is required; Gary Chanan showed that the Keck solution could be readily extrapolated to several thousands of segments [9]. Alternative methods have been proposed [10] and will be tested onsky in the forthcoming years. In the case of Owl, independent calibration of the primary and secondary mirrors is an added complexity, which favors in-pupil phasing sensors and subsequent Fourier filtering to disentangle the individual segmentation patterns.

Field stabilization and active optics are essentially based on the VLT concept, with the added complexity of controlling not one but two actively deformable surfaces (the tertiary and quaternary mirrors). This requires several guide stars, in theory three and in practice five to seven, and a corresponding number of wavefront sensors. Sky coverage is not an issue in view of the low spatial sampling required, hence limiting source magnitude $(\mathrm{v} \sim 18-20)$ and of the available field 
of view (10 arc minutes). It should be noted that the availability of two deformable surfaces provides for extended correction capability, no longer limited to the field position of a unique wavefront sensor.

Adaptive optics (AO) undoubtedly constitutes the most daring challenge. It is also absolutely essential; seeing limited observations with a 100-m class telescope would imply impossibly short focal ratio of the instrumentation, and immediate saturation by the sky background. A smaller on-sky resolution element is the only way out, which implies at least some degree of adaptive compensation for atmospheric turbulence.

\begin{tabular}{|c|c|c|}
\hline \multicolumn{2}{|c|}{ Wavefront control function } & Objective, metrology and active elements \\
\hline $\begin{array}{l}\text { Pre-setting } \\
\text { Metrology: } \\
\text { Correction: }\end{array}$ & $\Rightarrow$ & $\begin{array}{l}\text { bring optical system into linear regime } \\
\text { internal, tolerances } \sim 1-2 \mathrm{~mm}, \sim 5 \text { arc secs } \\
\text { re-position Corrector, M3 / M4 / M5 }\end{array}$ \\
\hline $\begin{array}{l}\text { Phasing } \\
\text { Metrology: } \\
\text { Correction: }\end{array}$ & $\Rightarrow$ & $\begin{array}{l}\text { keep } M 1 \text { and } M 2 \text { phased within tolerances } \\
\text { Edge sensors, Phasing WFS } \\
\text { Segments actuators }\end{array}$ \\
\hline $\begin{array}{l}\text { Field Stabilization } \\
\text { Metrology: } \\
\text { Correction: }\end{array}$ & $\Rightarrow$ & $\begin{array}{l}\text { cancel "fast" image motion } \\
\text { Guide probe } \\
\text { M6 tip-tilt (flat, exit pupil, } 2.35-\mathrm{m} \text { ) }\end{array}$ \\
\hline $\begin{array}{l}\text { Active optics } \\
\text { Metrology: } \\
\text { Correction: }\end{array}$ & $\begin{array}{l}\Rightarrow \\
\Rightarrow\end{array}$ & $\begin{array}{l}\text { finish off alignment / collimation } \\
\text { relax tolerances, control performance \& prescription } \\
\text { Wavefront sensor(s) } \\
\text { Rotation \& piston M5; M3 \& M4 active deformations }\end{array}$ \\
\hline $\begin{array}{l}\text { Adaptive optics } \\
\text { Metrology: } \\
\text { Correction: }\end{array}$ & $\Rightarrow$ & $\begin{array}{l}\text { atmospheric turbulence, residuals } \\
\text { Wavefront sensor(s) } \\
\text { M5, M6, ... }\end{array}$ \\
\hline
\end{tabular}

Table 2. Outline of Owl wavefront control functions.

The most advanced astronomical AO systems today pack around 700 degrees of freedom and their efficiency in normal operation is still to be assessed; although there are promising avenues for development, the implementation plan for an Owtclass AO facility must follow a careful, gradual path. The first stage will be implemented in the sixth mirror (M6, diameter $2.5-\mathrm{m})$ and provide for ground-layer correction ${ }^{9}$ or classical AO with natural guide stars in the infrared. Today's technology would allow for $\sim 1.8 \mathrm{~mm}$ thick, $2 \mathrm{~m}$ class mirror shells and an actuator interspacing of $\sim 30 \mathrm{~mm}$. For Owl first AO stage, the likely requirements become $\sim 2.5-\mathrm{m}$ diameter, $1 \mathrm{~mm}$ thick (possibly less), and $10-15 \mathrm{~mm}$ actuator interspacing. Although far from trivial, the implied technological development over a decade is incremental and does not seem unreasonably challenging. Moderate segmentation of the in-pupil AO mirror is a backup solution. Wavefront sensing will require large format $(512 \times 512)$, fast, low read-out noise detectors which also represent a challenge, albeit moderate: experts consider a 256x256 detector as probably feasible today. On the positive side, the large aperture will probably allow for a better sky coverage with Natural Guide Stars (NGS). The reason is purely geometrical: with a small aperture, quite a number of guide stars are needed to probe the volume of turbulence across the field of view. With a sufficiently large aperture, this number should decrease as the volume probed with individual guide stars in relation to the total volume to be probed increases. Simulations indicate that in the K band, a sky coverage of about $30 \%$ down to the galactic pole may be possible.

For extreme AO (high level correction), Laser Guide Stars (LGS) will most likely be required. Even though the underlying science cases will probably imply rather small field of view, several LGS are needed to cope with the cone effect. In addition, the LGS being at finite distance, their imaging through the telescope does not provide for sufficient image quality, even after refocusing them. In brief, imaging a source at $90 \mathrm{~km}$ with Owl is doing macro-photography, something it can hardly be designed for. It has been shown that NGS will still be required to sense the lowest wavefront modes [8] but the implied limitation in terms of field coverage are comparable to those applying to 8- to 10-m class

\footnotetext{
${ }^{9}$ i.e. relatively wide-field seeing reduction to $\sim 0.2$ arc seconds, depending on turbulence intensity and structure.
} 
telescope for the sensing of wavefront tilt. Alternative ways to do wavefront sensing on LGS are being explored by Ragazzoni et al, but at this day no proof-of-concept is yet available ${ }^{10}$.

Although a clear objective, extreme $\mathrm{AO}$ at visible wavelength will certainly require more technology development, delaying its implementation to later stages. MOEMS (Micro-Opto-Electronical Mechanical devices) constitute a promising avenue, however not the only one. Piezostacks, combined with thin shells and moderate segmentation in a pupil, may offer a more conservative baseline.

In conclusion, adaptive optics is the only control system that still requires extensive but not unreasonable technology development. The project is caut iously allocating more than $10 \%$ of its total budget to adaptive optics. Last but not least, an aptly named MAD (Multi-conjugate Adaptive optics Demonstrator) instrument is currently under construction at ESO to demonstrate the viability of relatively wide-field AO correction [11]. On-sky results are expected early 2005 and will plausibly constitute a go-no-go milestone for Owl.

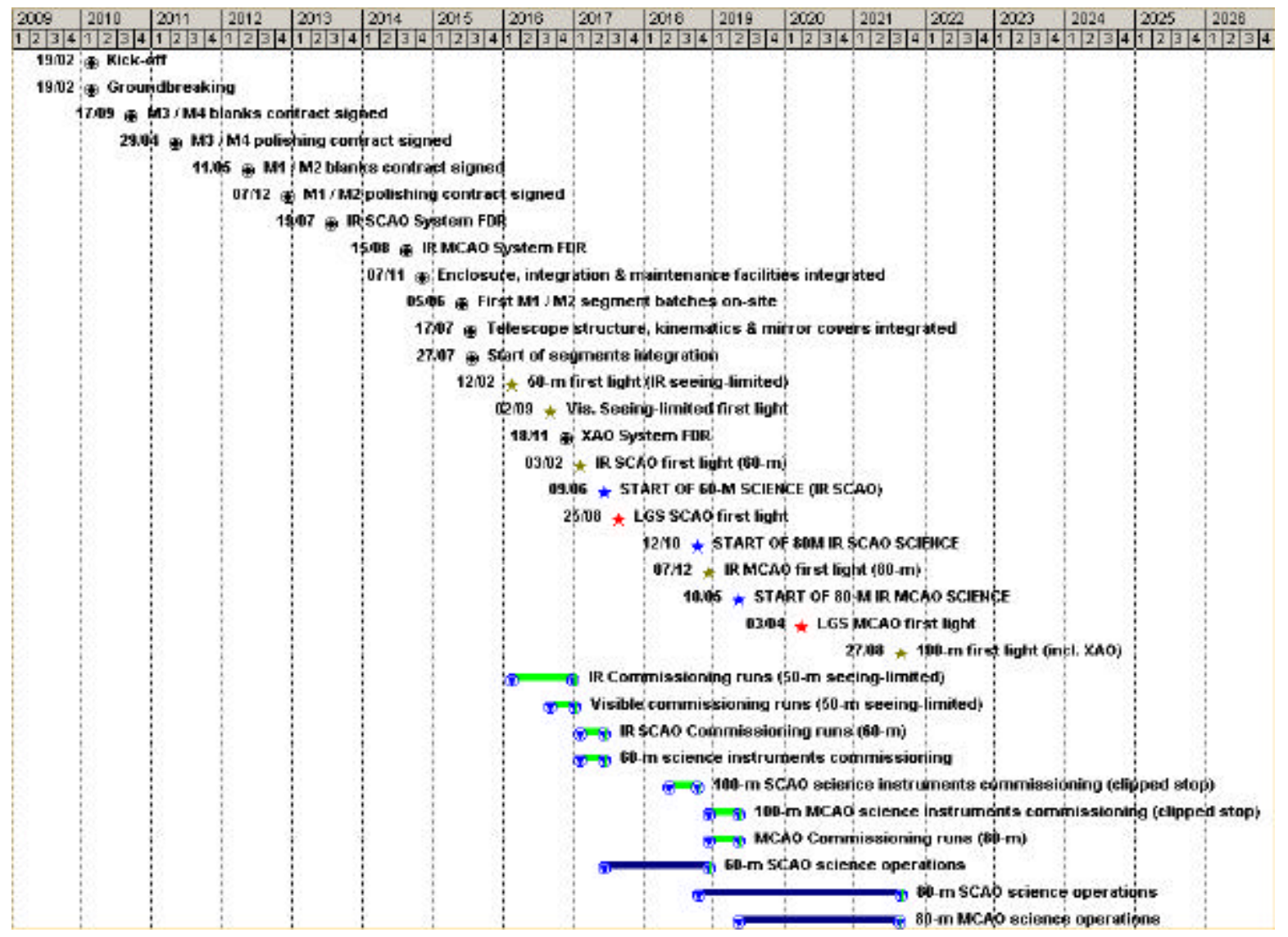

Fig. 5. Schedule estimate

\footnotetext{
${ }^{10}$ The idea is to select light beams coming from the elongated LGS in such a way that taken individually, they have the same properties as if they were coming from an infinite distance, as seen by the wavefront sensor. The concept is called PIGS for Pseudo-Infinite Guide Stars and should be tested on-sky in 2003.
} 


\section{COST AND SCHEDULE ESTIMATES}

Cost and schedule estimates for Owl are periodically updated with the progress of the design, analysis, and industrial studies. So far these studies have unequivocally proven the enormous benefits of a design favorable to industrialized, serial- or mass-production processes. The current estimate is 940 million Euros in capital investment for final design and construction. Roughly half of that figure is already supported by industrial studies, and a few more are planned to consolidate the final estimate. It assumes the eventual site to be at moderate altitude, to have low seismicity, low average wind speed, favorable soil properties, and to be reasonably close to adequate, pre-existing infrastructures. Probably none of these factors are required to ensure feasibility, but each could have a significant cost impact.

Elaborate schedule estimates have been drawn on the basis of industrial studies and past experience. As the aperture will be gradually filled, science operation may start ahead of the full completion date. According to current plans, commissioning could be completed with provisional, non-adaptive M5 and M6 units (see figure 1 for the numbering of the surfaces). Once the aperture would be filled over a diameter of $60 \mathrm{~m}$, a provisional, passive M6 unit could be replaced by the final, adaptive one, thereby allowing an early start of science with near-IR, with classical adaptive optics capability and wide-field seeing reduction. The second stage of adaptive optics (M5) would be integrated with the aperture reaching $80-\mathrm{m}$. Figure 5 shows a possible final design and construction schedule allowing a start of science operations in 2017. It is based on the assumption that an extensive phase B would be conducted over the period 2006 2010 before obtaining full funding for construction. If funding were readily available at the time of writing of this article, first light could occur in 2012. To some extent the schedule shown in figure 5 has been optimized to streamline cash-flow requirements, and to maximize R\&D time for the AO modules. It also includes more than a year engineering time before the telescope is handed over to the scientific community. Again, site properties may have a significant impact; the assumptions are consistent with those underlying the cost estimates.

\section{REFERENCES}

1. R. Gilmozzi, B. Delabre, P. Dierickx, N. Hubin , F. Koch, G. Monnet, M. Quattri, F. Rigaut, R.N. Wilson, The Future of Filled Aperture Telescopes: is a 100m Feasible?; 1998, Advanced Technology Optical/IR Telescopes VI, SPIE 3352, 778

2. A. B. Meinel, An overview of the Technological Possibilities of Future Telescopes, 1978, ESO Conf. Proc. 23, 13.

3. L. D. Barr, Factors Influencing Selection of a Next Generation Telescope Concept, 1979, Proc. SPIE Vol. $172,8$.

4. A. Ardeberg, T. Andersen, B. Lindberg, M. Owner-Petersen, T. Korhonen, P. Søndergård, Breaking the $8 m$ Barrier - One Approach for a 25m Class Optical Telescope, ESO Conf. And Workshop Proc. No 42, pp. 75-78, 1992.

5. M. Mountain, What is beyond the current generation of ground-based 8-m to 10-m class telescopes and the VLT-I?, SPIE 2871, pp. 597-606, 1996.

6. P. Dierickx, B. Delabre, L. Noethe, OWL optical design, active optics and error budget; Proc.SPIE, 4003, 2000.

7. P. Dierickx, J. Beletic, B. Delabre, M. Ferrari, R. Gilmozzi, N. Hubin, The Optics of the OWL 100-M Adaptive Telescope; 2000, Proceedings Bäckaskog Workshop on Extremely Large Telescopes, p97.

8. P. Dierickx, Optical design and adaptive optics properties of the OWL 100-m telescope; Proc. ESO Conf. Beyond Conventional Adaptive Optics, Venice, 2001, (http://lenin.pd.astro.it/venice2001/proceedings/).

9. G.Chanan, M.troy, C.Ohara, "Phasing the primary mirror segments of the Keck telescopes: a comparison of different techniques", Proc.SPIE, 4003, 188-201,2000.

10. A. Schumacher, L. Montoya, N. Devaney, K. Dohlen, P. Dierickx, Phasing ELTs for Adaptive Optics: preliminary results of a comparison of techniques, Proc. ESO Conf. Beyond Conventional Adaptive Optics, Venice, 2001 (http://lenin.pdastro.it/venice2001/proceedings).

11. E. Marchetti, N. Hubin, E. Fedrigo, J. Brynnel, B. Delabre, R. Donaldson, F. Franza, R. Conan, M. Le Louarn, C. Cavadore, A. Balestra, D. Baade, J. L. Lizon, R. Gilmozzi, G. Monnet, R. Ragazzoni, C. Arcidiacono, A. Baruffolo, E. Diolaiti, J. Farinato, E. Viard, D. Butler, S. Hippler, A. Amorim; MAD the ESO multi-conjugate adaptive optics demonstrator; 2002, Proc SPIE conf. Adaptive Optical System Technologies II. 